\title{
Application of Glycine, Folic Acid, and Moringa Extract as Bio-stimulants for Enhancing the Production of 'Flame Seedless' Grape Cultivar
}

\author{
Walid F. A. Mosa, ${ }^{\mathrm{a}, *}$ Mohamed Z. M. Salem, ${ }^{\mathrm{b}, * *}$ Asma A. Al-Huqail, ${ }^{\mathrm{c}, * * *}$ and \\ Hayssam M. Ali ${ }^{\mathrm{c}}$
}

\begin{abstract}
In this study, 130 uniform 'Flame Seedless' grape trees were selected for and subjected to the same cultural practices. The trees were sprayed three times, before flowering, during full bloom, and three weeks later with the following treatments: control (water only), 250, 500, and $750 \mathrm{ppm}$ glycine; 50,100 , and $150 \mathrm{ppm}$ folic acid (FA); $2 \%, 4 \%$, and $6 \%$ leaf moringa aqueous extract (MLAE); and their combinations. High-performance liquid chromatography (HPLC) analysis of moringa leaf aqueous extract (MLAE) showed the presence of the phenolic compounds ellagic acid, vanillic acid, p-hydroxy benzoic acid, catechol, and gallic acid with values of 54.18, $18.79,14,12.32$, and $12.12 \mathrm{mg} / 100 \mathrm{~g}$, respectively. The obtained results showed that the foliar spraying of 250, 500 , and $750 \mathrm{ppm}$ glycine, $4 \%$ and $6 \% \mathrm{MLAE}$, and their combinations of glycine $500 \mathrm{ppm}+\mathrm{FA} 100 \mathrm{ppm}+$ MLAE 4\% and glycine 750 ppm + FA 150 ppm + MLAE 6\% significantly increased the shoot length, shoot thickness, leaf chlorophyll content, yield, and fruit quality over the control. Glycine at $750 \mathrm{ppm}$ was the best treatment followed by glycine at $500 \mathrm{ppm}$ compared with the other applied treatments and the control in both experimental seasons.
\end{abstract}

Keywords: Moringa extract; Glycine; Folic acid; Grape; Fruit quality

Contact information: a: Plant Production Department (Horticulture- Pomology), Faculty of Agriculture, Saba Basha, Alexandria University, Alexandria 21531 Egypt; b: Forestry and Wood Technology Department, Faculty of Agriculture (El-Shatby), Alexandria University, Alexandria 21545 Egypt;

c: Chair of Climate Change, Environmental Development and Vegetation Cover, Department of Botany and Microbiology, College of Science, King Saud University, Riyadh 11451, Saudi Arabia;

Corresp.authors: *walidbreeder@yahoo.com; **zidan_forest@yahoo.com; ***aalhuqail@ksu.edu.sa

\section{INTRODUCTION}

Grape (Vitis vinifera L.) is a member of the Vitaceae family. In Egypt, the harvested area is 78853 ha, which produces 1759472 tons. It was stated by many authors that berries of grape have been utilized as table fruit, juice, and for the production of wine, and raisins, as well as in the industries of cosmetic from leaf, seed, and skin extracts (Iriti and Faoro 2006; Monagas et al. 2006). Moreover, grape skin, pulp, and seeds could be used to produce wine, terpenes, and norisoprenoids, as well as sugars, which could be converted to alcohol (Lund and Bohlmann 2006). It was reported by (Ruberto et al. 2007; Hogan et al. 2010; Drosou et al. 2015) that grapes are characterized by the high content of phenolic compounds, which are a valuable source of natural antioxidants. Grapes are a crucial exporter to potassium and manganese (Torres-Urrutia et al. 2011), rich in short-chain carbohydrates (Gibson and Shepherd 2012; Gibson 2017), and vitamin C (Moores 2013; Carr and Maggini 2017), which is important for immunology. 
Glycine is an amino acid that has a crucial role in improving total chlorophyll and vegetative growth in grapes, as well as in increasing the availability of $\mathrm{Fe}, \mathrm{Zn}, \mathrm{Mn}$, and $\mathrm{Cu}$ to the plants (Sekhon 2003; Liu et al. 2011; Ghasemi et al. 2013; Shan et al. 2016; Razavi et al. 2018). L-glycine works as a signal transduction molecule that contributes to making plant nutrients readily available (Teixeira et al. 2017). The plant height, leaf chlorophyll content, and fresh weight of the shoot and root of Coriandrum sativum were significantly improved by the application of glycine, especially at higher concentrations (Souri and Hatamian 2019). In addition, the application of glycine increased the total soluble solid and vitamin $\mathrm{C}$ contents compared with that in the controls. In comparison with the unfertilized plants, the leaf mineral contents of $\mathrm{N}, \mathrm{Ca}, \mathrm{K}, \mathrm{P}, \mathrm{Fe}$, and $\mathrm{Zn}$ were significantly increased by the application of glycine (Jiang et al. 2020; Lo'ay and Doaa 2020).

Folic acid (FA) has a fundamental role in the metabolism of amino acids and in nucleic acids synthesis (Paucean et al. 2018). Moreover, FA was found to increase the productivity of Pisum sativum by affecting the leaf content of chlorophyll, as well as the yield, weight, and quality of the seed (Burguieres et al. 2007). Foliar spraying of sweet pepper plants with FA increased flowering, yield, and fruit quality (Al-Said and Kamal 2008). Additionally, exogenous FA showed a positive effect on the growth, yield, and quality of soybean and strawberry plants (Mansour 2014; Li et al. 2015). Spraying of FA at $0,10,20$, and $30 \mathrm{mg} / \mathrm{L}$ on Luz de otono bean cultivar was investigated by Al-Maliky et al. (2019). The obtained results showed that spraying FA raised the height of plant, number of branches, leaf area, and shoot dry weight, as well as length, number and green weight of pods as compared to control. Moreover, 100 seeds weight, the production of green pods, and fresh seeds, the percentage of TSS, and dry matter, as well as protein and vitamin C and the concentration of $30 \mathrm{mg} / \mathrm{L}$ was the best concentration. Spraying FA at $150 \mu \mathrm{M} / \mathrm{L}$ under lack of water levels raised significantly plant growth, efficacy of water use, firmness of cell membrane, yield, total soluble solids, and protein content in snap beans (Phaseolus vulgaris L.) (Ibrahim et al. 2021).

Moringa oleifera contains around forty sex antioxidants such as ascorbate, carotenoids, phenols, and flavonoid (Iqbal and Bhanger 2006). Moringa leaf aqueous extract (MLAE) can be used as a bio-stimulant and contains macro- and micronutrients, amino acids, ascorbic acids, minerals, and growth-enhancing principles such as the hormone of the cytokinin type (Makkar et al. 2007). In addition, the growth hormone spray will cause the plants to be firmer and more resistant to pest and diseases. Moreover, $M$. oleifera is one of the best crop treatments with confirmed impacts on growth and yield, and it can be used by farmers as a source for nutrients instead of relying on inorganic fertilizers, which are costly and are associated with both land and soil degradation and environmental pollution (Phiri 2010). Additionally, M. oleifera is standardized to contain flavonoid, phenolic, and carotenoid, which can be used as antioxidants, one of them being quercetin (Alhakmani et al. 2013; Wang et al. 2017). Abbassy et al. (2020) reported that M. oleifera leaf extract is rich in prime metabolic compounds such as proteins, lipids, carbohydrates, minerals, vitamins, and amino acids. Moringa provides products that improve the growth and yield of various crops (Mohamed et al. 2020). Hollywood plum trees sprayed with 6\% MLAE had an improved fruit set, fruit yield, fruit weight, firmness, color, total soluble solids, vitamin $\mathrm{C}$, and the content of anthocyanin in the plum cultivar Hollywood, while they had reduced fruit drop compared to the use of 0\%, 4\%, or 5\% MLAE (Shaaban et al. 2020). 
Therefore, the objective of this study was to investigate the impacts of the foliar application of glycine (amino acid), folic acid, and MLAE as natural biostimulants to vegetative growth, yield, and fruit quality of the grape cv. 'Flame Seedless' to minimize the dependency on the chemical fertilization in grape orchards.

\section{EXPERIMENTAL}

\section{Preparation of MLAE and HPLC Analysis of Phytochemical}

Moringa oleifera Lam. leaves were obtained from Alexandria, Egypt and were shade-dried. The dried leaves were ground to powder using a small laboratory mill. Then, $100 \mathrm{~g}$ of the powdered leaves $(200 \mathrm{~g})$ were soaked in $2 \mathrm{~L}$ of distilled water for $24 \mathrm{~h}$ at room temperature (Mohamed et al. 2020). The mixture was filtered using Whatman No. 1 filter paper. The dissolved MLAE was concentrated by evaporation, then prepared at the concentrations 2\%, 4\%, and 6\%. An Agilent 1260 Infinity 1260 II LC, System (HPLC Agilent, Santa Clara, CA, USA) was equipped with a Quaternary pump and a Zorbax Eclipse Plus C18 column (100 mm × $4.6 \mathrm{~mm}$ i.d.) (Agilent Technologies, Santa Clara, CA, USA) and operated at $30^{\circ} \mathrm{C}$; it was used to identify the phytochemical compounds in the MLAE. Separation conditions and standard phenolic compounds can be found in previously published works (Al-Huqail et al. 2019; Behiry et al. 2019; Salem et al. 2019; Ashmawy et al. 2020; Salem et al. 2020).

\section{Experimental Location and Treatments}

This experiment was carried out during two successive seasons, in 2018 and 2019, on 10-year-old 'Flame Seedless' grape trees ( $V$. vinifera L.), planted with $3 \mathrm{~m}$ between rows and $2 \mathrm{~m}$ between trees in the same row, and grown in a calcareous soil under a drip irrigation system in a private orchard located at Nubaria, Beheira Governorate, Egypt. Physiochemical analysis of the experimental soil was performed (Table 1) (Sparks et al. 2020).

Table 1. Physical and Chemical Properties of the Experiment Soil

\begin{tabular}{|c|c|c|c|c|c|c|c|c|c|}
\hline $\begin{array}{l}\text { Soil Depth } \\
\text { (cm) }\end{array}$ & Texture & $\mathrm{pH}$ & $\begin{array}{l}{ }^{*} E C \\
(d S / m)\end{array}$ & $\mathrm{N}(\%)$ & $\mathrm{P}(\%)$ & $\mathrm{K}(\%)$ & $\begin{array}{c}\mathrm{Fe} \\
(\mathrm{mg} / \mathrm{L})\end{array}$ & $\begin{array}{c}\mathrm{Zn} \\
(\mathrm{mg} / \mathrm{L})\end{array}$ & $\begin{array}{c}\mathrm{Mn} \\
(\mathrm{mg} / \mathrm{L})\end{array}$ \\
\hline \multirow{4}{*}{0 to 60} & $\begin{array}{l}\text { Sandy } \\
\text { loam }\end{array}$ & 8.6 & 2.22 & 16.62 & 23.20 & 30.25 & 0.35 & 0.10 & 0.08 \\
\hline & \multirow{2}{*}{\multicolumn{2}{|c|}{$\mathrm{CaCO}_{3}(\mathrm{mg} / \mathrm{L})$}} & \multicolumn{4}{|c|}{ Cations (meq/100 g Soil) } & \multirow{2}{*}{$\begin{array}{l}\text { O.M. } \\
(\%)\end{array}$} & \multirow{2}{*}{$\mathrm{Cl}^{-}$} & \multirow{2}{*}{$\mathrm{HCO}_{3}^{-}$} \\
\hline & & & $\mathrm{Na}^{+}$ & $\mathrm{K}^{+}$ & $\mathrm{Ca}_{2}^{+}$ & $\mathrm{Mg}^{++}$ & & & \\
\hline & \multicolumn{2}{|c|}{28.42} & 10.18 & 1.12 & 6.52 & 3.63 & 0.53 & 12.3 & 6.2 \\
\hline
\end{tabular}

A total of 130 uniform trees were selected for this study, and all of them were subjected to the same cultural practices in the two seasons. They were sprayed with the experimental treatments three times in each season: before flowering, during full bloom, and three weeks later (Table 2). These applied treatments were arranged in a randomized complete block design where each treatment was composed of 10 replicates. 
Table 2. Treatments Used for Spraying the Trees

\begin{tabular}{|c|c|}
\hline Treatment & Concentrations \\
\hline Control & Water only \\
\hline Glycine & 250,500, and $750 \mathrm{ppm}$ \\
\hline Folic acid (FA) & 50,100, and $150 \mathrm{ppm}$ \\
\hline MLAE & $2 \%, 4 \%$, and $6 \%$ \\
\hline Combination 1 & $250 \mathrm{ppm}$ glycine $+50 \mathrm{ppm} \mathrm{FA}+2 \% \mathrm{MLAE}$ \\
\hline Combination 2 & $500 \mathrm{ppm}$ glycine $+100 \mathrm{ppm} \mathrm{FA}+4 \% \mathrm{MLAE}$ \\
\hline Combination 3 & $750 \mathrm{ppm}$ glycine $+150 \mathrm{ppm} \mathrm{FA}+6 \% \mathrm{MLAE}$ \\
\hline
\end{tabular}

\section{Measurement of Vegetative Parameters, Leaf Total Chlorophyll, Fruit Yield, Fruit Quality and Leaf Chemical Composition}

Shoot length and thickness $(\mathrm{cm})$ were measured at the end of each growing season. Total chlorophyll in the fresh leaves was determined with SPAD units as evaluated by a chlorophyll meter (SPAD-502; Konica Minolta, Osaka, Japan). The number of clusters per each vine and weight of each cluster was measured and recorded. The yield of vine $(\mathrm{kg})$ was determined by the weight of clusters per vine multiplied by the number of clusters per vine. Yield in tons per hectare was estimated by multiplying yield of vine $\times$ number of vines per hectare.

For the leaf chemical composition, samples of 30 leaves were taken from the middle of the shoots and were randomly selected from each replicate after the harvest time in June to determine their content in terms of percentages of nitrogen $(\mathrm{N})$, phosphorus $(\mathrm{P})$, and potassium (K) (Arrobas et al. 2018). The leaf samples were washed first with tap water and then with distilled water and dried at $70{ }^{\circ} \mathrm{C}$ until a constant weight was obtained. Finally, the dried leaf samples were ground and acid digested using $\mathrm{H}_{2} \mathrm{SO}_{4}$ and $\mathrm{H}_{2} \mathrm{O}_{2}$ until the digested solution became clear. The digested solution was used for the determination of nitrogen using the micro-Kjeldahl method (Wang et al. 2016), phosphorus by the vanadomolybdate method (Weiwei et al. 2017), and potassium using a flame photometer (Banerjee and Prasad 2020).

\section{Measuring of Fruit Quality in terms of Physical, and Chemical Characteristics}

At the time of harvesting, 10 clusters from each vine/replicate were chosen randomly to determine their physical and chemical characteristics; cluster weight (g), size $\left(\mathrm{cm}^{3}\right)$, length $(\mathrm{cm})$, and width $(\mathrm{cm})$. In addition, 100 berries were selected from all the chosen clusters of each vine to measure their weight $(\mathrm{g})$, size $\left(\mathrm{cm}^{3}\right)$, juice $(\%)$, berry weight $(\mathrm{g})$, length $(\mathrm{mm})$, and width $(\mathrm{mm})$. Fruit firmness $\left({\left.\mathrm{lb} / \mathrm{in}^{2}\right)}^{2}\right.$ using a Magness-Taylor pressure tester (mod. FT 02 (0-2 Lb., Via Reale, 63 - 48011 Alfonsine, Italy) with a 1/16-in plunger. Total soluble solids (TSS) were determined using a hand-held refractometer (ATAGO N2E Brix 28-62\% made in Japan) and the results were expressed as percentages (\%). The percentage of titratable acidity in the fruit juice of 100 berries was determined using the method described by Turner et al. (2011). Total sugars were determined calorimetrically using Nelson arsenate-molybdate colorimetric method (Nielsen 2010). Anthocyanin was determined at the stage of coloration (mg/100 g fresh weight peel) (Nangle et al. 2015).

\section{Statistical Analysis}

The obtained data were subjected to one-way analysis of variance (Ott and Longnecker 2015). A least significant difference at $0.05 \%$ was used to compare the means 
of the treatments and measured with CoHort Software (Pacific Grove, CA, USA).

\section{RESULTS AND DISCUSSION}

\section{HPLC Analysis of Polyphenols of MLAE}

Figure 1 shows the separation chromatograms of the identified chemical compounds in MLAE. Table 3 shows that ellagic acid (54.18), vanillic acid (18.79), phydroxy benzoic acid (14), catechol (12.32), and gallic acid (12.12) were the main identified phenolic compounds.

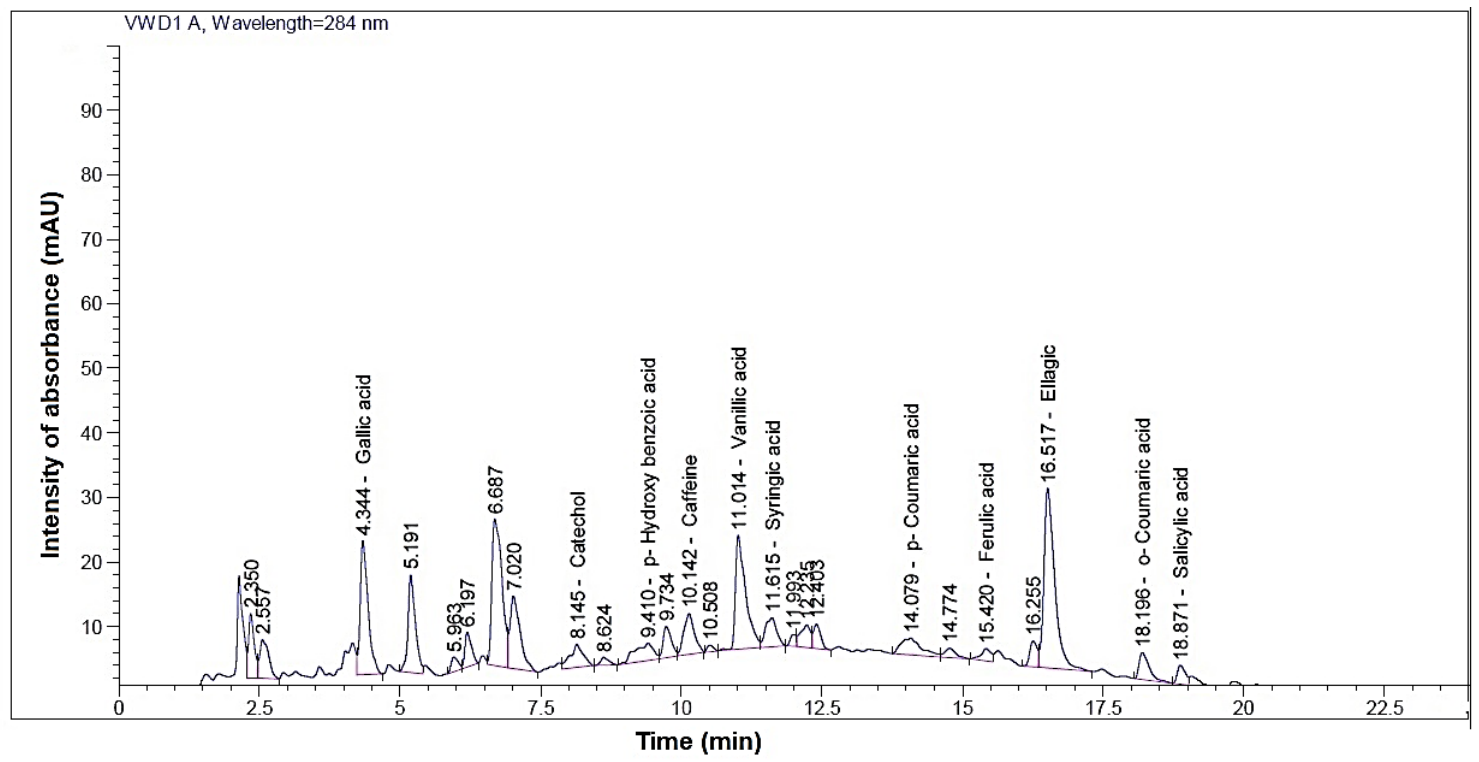

Fig. 1. HPLC chromatogram of phenolic compounds and caffeine identified in MLAE

Table 3. Identified Chemical Composition of Phenolic Compounds and Caffeine of MLAE by HPLC

\begin{tabular}{|c|c|}
\hline Compound & Concentration $(\mathrm{mg} / 100 \mathrm{~g})$ \\
\hline Gallic acid & 12.12 \\
\hline Catechol & 12.32 \\
\hline p-Hydroxy benzoic acid & 14 \\
\hline Caffeine & 6.57 \\
\hline Vanillic acid & 18.79 \\
\hline Caffeic acid & ND * \\
\hline Syringic acid & 3.69 \\
\hline Vanillin & ND \\
\hline p-Coumaric acid & 1.93 \\
\hline Ferulic acid & 1.20 \\
\hline Ellagic acid & 54.18 \\
\hline Benzoic acid & ND \\
\hline o-Coumaric acid & 2.37 \\
\hline Salicylic acid & 4.70 \\
\hline Cinnamic acid & ND \\
\hline
\end{tabular}




\section{Vegetative Parameters}

Table 4 shows that the shoot length significantly increased as a consequence of the foliar spraying with glycine at $750 \mathrm{ppm}$, which gave the highest increment compared to the control in both seasons. In addition, it was enhanced by the foliar spraying of glycine at 250 and $500 \mathrm{ppm}$, MLAE at 4 and $6 \%$ and with the combinations of glycine $750 \mathrm{ppm}+$ FA $150 \mathrm{ppm}+6 \%$ MLAE and glycine $500 \mathrm{ppm}+\mathrm{FA} 100 \mathrm{ppm}+$ MLAE 4\% in the two seasons as compared with the control. Compared with the control in both seasons, the shoot thickness was significantly enhanced by the applications of glycine at 250, 500, and 750 ppm, MLAE at 2\%, 4\%, and 6\%, and FA at 50, 100, and $150 \mathrm{ppm}$. Additionally, it improved with the combinations of glycine $250 \mathrm{ppm}+$ FA $50 \mathrm{ppm}+2 \%$ MLAE, glycine $500 \mathrm{ppm}+\mathrm{FA} 100 \mathrm{ppm}+\mathrm{MLAE} 4 \%$, and glycine $750 \mathrm{ppm}+$ FA $150 \mathrm{ppm}+$ MLAE 6\%. The total chlorophyll content in the leaves was greatly improved by the spraying with glycine at 250, 500, and $750 \mathrm{ppm}$, with MLAE at 2, 4, and 6\%, and with FA at 100 and $150 \mathrm{ppm}$. In addition, it was raised with the combinations of glycine $250 \mathrm{ppm}+$ FA $50 \mathrm{ppm}$ + MLAE 2\%, glycine $500 \mathrm{ppm}+$ FA $100 \mathrm{ppm}+4 \%$ MLAE, and glycine $750 \mathrm{ppm}+\mathrm{FA}$ $150 \mathrm{ppm}+6 \%$ MLAE to a higher extent than the control in both experimental seasons. The best results were obtained by the foliar spraying of glycine at $750 \mathrm{ppm}$ over the other applied treatments or the control in the two seasons.

Table 4. Influence of the Foliar Application of Glycine, FA, MLAE, and their Combinations on Shoot Length, Thickness, and Total Chlorophyll of Grape cv.

'Flame Seedless' During 2018 and 2019 Seasons

\begin{tabular}{|c|c|c|c|c|c|c|}
\hline \multirow{2}{*}{ Treatment } & \multicolumn{2}{|c|}{ Shoot Length $(\mathrm{cm})$} & \multicolumn{2}{|c|}{$\begin{array}{l}\text { Shoot Thickness } \\
(\mathrm{mm})\end{array}$} & \multicolumn{2}{|c|}{$\begin{array}{l}\text { Total Chlorophyll } \\
\text { SPAD }\left(\mu \mathrm{Molm}^{-2}\right)\end{array}$} \\
\hline & 2018 & 2019 & 2018 & 2019 & 2018 & 2019 \\
\hline Control & $96.00 \mathrm{~b}$ & $96.80 d$ & $7.82 d$ & $8.20 \mathrm{~h}$ & $31.68 c$ & $32.69 \mathrm{e}$ \\
\hline Glycine 250 ppm & $98.60 a b$ & 100.00abc & $8.96 b c$ & 9.98abc & $38.60 a$ & $39.44 a b$ \\
\hline Glycine 500 ppm & $98.80 \mathrm{ab}$ & $101.40 \mathrm{ab}$ & $9.78 a$ & $10.04 a b$ & $38.86 a$ & $39.66 a$ \\
\hline Glycine 750 ppm & $100.60 \mathrm{a}$ & $101.80 \mathrm{a}$ & $10.12 a$ & $10.30 \mathrm{a}$ & $39.72 a$ & $40.14 a$ \\
\hline FA 50 ppm & $96.00 \mathrm{~b}$ & $98.40 \mathrm{~cd}$ & $8.52 \mathrm{c}$ & $8.90 \mathrm{~g}$ & $32.78 c$ & $33.70 \mathrm{e}$ \\
\hline FA 100 ppm & $97.40 a b$ & $98.60 \mathrm{bcd}$ & $8.56 c$ & $8.96 f g$ & $34.74 b$ & $35.76 \mathrm{~d}$ \\
\hline FA 150 ppm & $98.20 a b$ & $99.20 \mathrm{abcd}$ & $8.64 \mathrm{C}$ & 9.34def & $35.69 b$ & $35.94 d$ \\
\hline MLAE $2 \%$ & 97.60ab & $100.40 \mathrm{abc}$ & $8.64 \mathrm{c}$ & $9.04 \mathrm{fg}$ & $34.82 \mathrm{~b}$ & $36.19 \mathrm{~cd}$ \\
\hline MLAE 4\% & $98.60 a b$ & 100.60abc & $8.94 b c$ & $9.52 \mathrm{de}$ & $38.38 a$ & $37.88 \mathrm{bc}$ \\
\hline MLAE 6\% & $98.60 \mathrm{ab}$ & $100.80 \mathrm{abc}$ & $9.16 b$ & $9.58 \mathrm{cde}$ & $38.49 a$ & $39.89 a$ \\
\hline $\begin{array}{l}\text { Glycine } 250 \text { ppm + FA } 50 \text { ppm } \\
+ \text { MLAE } 2 \%\end{array}$ & 98.20ab & $99.60 \mathrm{abcd}$ & $8.98 \mathrm{bc}$ & $9.24 \mathrm{efg}$ & $35.69 b$ & $35.86 d$ \\
\hline $\begin{array}{l}\text { Glycine } 500 \text { ppm + FA } 100 \\
\text { ppm + MLAE 4\% }\end{array}$ & 98.60ab & $101.00 \mathrm{abc}$ & $9.14 b$ & $9.74 \mathrm{bcd}$ & $38.91 a$ & $38.77 a b$ \\
\hline $\begin{array}{l}\text { Glycine } 750 \text { ppm + FA } 150 \\
\text { ppm + MLAE 6\% }\end{array}$ & 98.80ab & 101.20abc & $9.80 a$ & 10.04ab & 39.09a & $39.96 a$ \\
\hline${ }^{*}$ LSD 0.05 & 3.47 & 2.90 & 0.48 & 0.40 & 1.53 & 1.74 \\
\hline
\end{tabular}

\section{Fruit Yield}

Table 5 demonstrates that the foliar spraying with glycine at 250, 500, and 750 ppm, FA at 50, 100, and $150 \mathrm{ppm}$, and MLAE at 2, 4, and 6\% significantly increased the 
clusters number, cluster weight, yield per vine $(\mathrm{kg})$ and yield in ton per hectare comparing with the control in the two seasons. Moreover, they also were statistically raised by the foliar application of glycine $250 \mathrm{ppm}+$ FA $50 \mathrm{ppm}+$ MLAE $2 \%$, glycine $500 \mathrm{ppm}+\mathrm{FA}$ $100 \mathrm{ppm}+$ MLAE 4\%, and glycine $750 \mathrm{ppm}+$ FA $150 \mathrm{ppm}+$ MLAE 6\% combinations over the control. The highest increment and the best results were obtained by the foliar spraying of glycine, especially at 750 and $500 \mathrm{ppm}$, respectively, compared with the other applied treatments and the control in both experimental seasons.

Table 5. Influence of the Foliar Application of Glycine, FA, MLAE, and Their Combinations on the Number and Weight of Clusters, Yield in $\mathrm{kg}$ per Vine and Yield in ton per Hectare of Grape cv. 'Flame Seedless' During 2018 and 2019 Seasons

\begin{tabular}{|c|c|c|c|c|c|c|c|c|}
\hline \multirow{2}{*}{ Treatment } & \multicolumn{2}{|c|}{ Clusters Number } & \multicolumn{2}{|c|}{ Cluster Weight (g) } & \multicolumn{2}{|c|}{ Yield (kg)/vine } & \multicolumn{2}{|c|}{ Yield (ton)/hectare } \\
\hline & 2018 & 2019 & 2018 & 2019 & 2018 & 2019 & 2018 & 2019 \\
\hline Control & $30.80 \mathrm{~h}$ & $32.60 i$ & $369.00 \mathrm{k}$ & $376.60 \mathrm{k}$ & $11.40 \mathrm{j}$ & 12.20j & $18.20 \mathrm{j}$ & 19.80j \\
\hline Glycine 250 ppm & $43.80 c$ & $45.20 c$ & $758.00 c$ & $772.00 \mathrm{c}$ & $33.20 \mathrm{c}$ & $35.00 \mathrm{c}$ & $53.00 \mathrm{c}$ & $55.80 \mathrm{c}$ \\
\hline Glycine 500 ppm & $45.40 \mathrm{~b}$ & $47.00 \mathrm{~b}$ & $772.80 b$ & $781.60 \mathrm{~b}$ & $35.20 \mathrm{~b}$ & $36.80 \mathrm{~b}$ & $56.20 \mathrm{~b}$ & $59.00 \mathrm{~b}$ \\
\hline Glycine 750 ppm & $47.60 \mathrm{a}$ & $50.80 a$ & $841.20 a$ & $846.00 a$ & $40.00 a$ & $43.20 a$ & $64.00 \mathrm{a}$ & $68.80 a$ \\
\hline FA $50 \mathrm{ppm}$ & $33.60 \mathrm{~g}$ & $35.00 \mathrm{~h}$ & $456.60 \mathrm{j}$ & 465.60j & $15.40 \mathrm{i}$ & $16.60 \mathrm{i}$ & $24.40 i$ & $26.20 \mathrm{i}$ \\
\hline FA 100 ppm & $34.40 \mathrm{fg}$ & $36.00 \mathrm{~h}$ & $459.20 \mathrm{j}$ & $467.20 \mathrm{j}$ & $15.80 \mathrm{i}$ & $16.80 \mathrm{i}$ & $25.40 \mathrm{i}$ & $26.80 \mathrm{i}$ \\
\hline FA 150 ppm & $35.60 f$ & $38.20 \mathrm{~g}$ & $511.00 i$ & $517.00 \mathrm{i}$ & $18.40 \mathrm{~h}$ & $19.80 \mathrm{~h}$ & $29.00 \mathrm{~h}$ & $31.40 \mathrm{~h}$ \\
\hline MLAE $2 \%$ & $40.00 \mathrm{e}$ & $40.60 f$ & $576.00 \mathrm{~h}$ & $590.20 \mathrm{~h}$ & $23.00 \mathrm{~g}$ & $23.80 \mathrm{~g}$ & $36.80 \mathrm{~g}$ & $38.40 \mathrm{~g}$ \\
\hline MLAE 4\% & $41.80 \mathrm{~d}$ & $42.40 \mathrm{e}$ & $654.60 \mathrm{~g}$ & $656.80 \mathrm{~g}$ & $27.20 f$ & 28.00ef & $43.60 f$ & $44.40 f$ \\
\hline MLAE 6\% & $43.60 \mathrm{c}$ & 43.20de & $665.20 f$ & $666.80 f$ & $29.00 \mathrm{e}$ & $28.80 \mathrm{e}$ & $46.20 \mathrm{e}$ & $46.40 \mathrm{e}$ \\
\hline $\begin{array}{l}\text { Glycine } 250 \text { ppm + } \\
\text { FA } 50 \text { ppm + MLAE } \\
2 \%\end{array}$ & $39.60 \mathrm{e}$ & $39.00 \mathrm{fg}$ & $707.00 \mathrm{e}$ & $705.80 \mathrm{e}$ & $27.80 f$ & $27.40 f$ & $44.60 f$ & $44.00 f$ \\
\hline $\begin{array}{l}\text { Glycine } 500 \text { ppm + } \\
\text { FA } 100 \text { ppm + } \\
\text { MLAE } 4 \%\end{array}$ & $42.60 \mathrm{~cd}$ & $43.80 \mathrm{cde}$ & $713.00 \mathrm{e}$ & $734.00 \mathrm{~d}$ & $30.60 d$ & $32.20 d$ & $48.60 d$ & $51.40 \mathrm{~d}$ \\
\hline $\begin{array}{l}\text { Glycine } 750 \text { ppm + } \\
\text { FA } 150 \text { ppm + } \\
\text { MLAE 6\% }\end{array}$ & $43.20 \mathrm{~cd}$ & $44.80 \mathrm{~cd}$ & $723.00 d$ & $736.00 \mathrm{~d}$ & $31.20 d$ & $33.20 d$ & $50.00 d$ & $52.80 \mathrm{~d}$ \\
\hline LSD 0.05 & 1.52 & 1.64 & 6.57 & 7.05 & 1.01 & 1.22 & 1.55 & 1.74 \\
\hline
\end{tabular}

\section{Fruit Physical and Chemical Characteristics}

Table 6 shows that the size, length, and width of the clusters had greatly increased by spraying glycine at 250,500, and $750 \mathrm{ppm}$ and by the combination of glycine $750 \mathrm{ppm}$ + FA $150 \mathrm{ppm}+$ MLAE 6\% compared with the control and the other applied treatments in both seasons. The application of glycine at $750 \mathrm{ppm}$ was superior and gave the best results in the two seasons rather than the other applied treatments. 
Table 6. Influence of the Foliar Application of Glycine, FA, MLAE, and Their Combinations on the Size, Length, and Width of Clusters of Grape cv. 'Flame Seedless' During 2018 and 2019 Seasons

\begin{tabular}{|l|c|c|c|c|c|c|}
\hline \multirow{2}{*}{ Treatment } & \multicolumn{2}{|c|}{ Cluster Size $\left(\mathrm{cm}^{3}\right)$} & \multicolumn{2}{l}{ Cluster Length (cm) } & \multicolumn{2}{l|}{ Cluster Width (cm) } \\
\cline { 2 - 7 } & 2018 & 2019 & 2018 & 2019 & 2018 & 2019 \\
\hline Control & $348.20 \mathrm{j}$ & $354.60 \mathrm{i}$ & $16.90 \mathrm{i}$ & $16.20 \mathrm{i}$ & $9.20 \mathrm{i}$ & $11.16 \mathrm{i}$ \\
\hline Glycine $250 \mathrm{ppm}$ & $738.20 \mathrm{c}$ & $755.60 \mathrm{~b}$ & $23.60 \mathrm{~b}$ & $25.20 \mathrm{c}$ & $13.48 \mathrm{c}$ & $14.60 \mathrm{c}$ \\
\hline Glycine 500 ppm & $753.20 \mathrm{~b}$ & $761.80 \mathrm{~b}$ & $23.90 \mathrm{~b}$ & $26.60 \mathrm{~b}$ & $14.40 \mathrm{ab}$ & $15.40 \mathrm{~b}$ \\
\hline Glycine 750 ppm & $821.60 \mathrm{a}$ & $824.80 \mathrm{a}$ & $25.90 \mathrm{a}$ & $30.80 \mathrm{a}$ & $14.77 \mathrm{a}$ & $16.60 \mathrm{a}$ \\
\hline FA 50 ppm & $435.00 \mathrm{i}$ & $448.20 \mathrm{~h}$ & $17.16 \mathrm{i}$ & $17.40 \mathrm{~h}$ & $10.40 \mathrm{~h}$ & $10.80 \mathrm{i}$ \\
\hline FA 100 ppm & $437.80 \mathrm{i}$ & $448.40 \mathrm{~h}$ & $17.70 \mathrm{hi}$ & $18.40 \mathrm{~h}$ & $10.40 \mathrm{~h}$ & $12.00 \mathrm{~h}$ \\
\hline FA 150 ppm & $489.40 \mathrm{~h}$ & $496.60 \mathrm{~g}$ & $18.60 \mathrm{gh}$ & $19.70 \mathrm{~g}$ & $11.10 \mathrm{gh}$ & $12.40 \mathrm{gh}$ \\
\hline MLAE 2\% & $555.20 \mathrm{~g}$ & $570.00 \mathrm{f}$ & $19.00 \mathrm{fg}$ & $20.80 \mathrm{f}$ & $11.50 \mathrm{fg}$ & $12.58 \mathrm{~g}$ \\
\hline MLAE 4\% & $637.00 \mathrm{f}$ & $635.80 \mathrm{e}$ & $19.80 \mathrm{ef}$ & $23.20 \mathrm{e}$ & $12.00 \mathrm{def}$ & $13.50 \mathrm{e}$ \\
\hline $\begin{array}{l}\text { MLAE 6\% } \\
\text { Flycine 250 ppm + FA 50 } \\
\text { ppm + MLAE 2\% }\end{array}$ & $642.80 \mathrm{f}$ & $642.60 \mathrm{e}$ & $21.60 \mathrm{~d}$ & $24.00 \mathrm{de}$ & $12.40 \mathrm{de}$ & $14.20 \mathrm{~cd}$ \\
\hline $\begin{array}{l}\text { Glycine 500 ppm + FA 100 } \\
\text { ppm + MLAE 4\% }\end{array}$ & $695.80 \mathrm{e}$ & $714.60 \mathrm{c}$ & $22.00 \mathrm{~cd}$ & $23.20 \mathrm{e}$ & $12.60 \mathrm{~d}$ & $14.08 \mathrm{~d}$ \\
\hline $\begin{array}{l}\text { Glycine 750 ppm + FA 150 } \\
\text { ppm + MLAE 6\% }\end{array}$ & $705.40 \mathrm{~d}$ & $716.00 \mathrm{c}$ & $23.00 \mathrm{bc}$ & $24.80 \mathrm{~cd}$ & $13.96 \mathrm{bc}$ & $15.40 \mathrm{~b}$ \\
\hline $\begin{array}{l}\text { LSD 0.05 } \\
\text { The difference between mean values shown on the same column with same letter is not significant } \\
\text { according to Duncan's multiple range test at P < 0.05. }\end{array}$ & 7.01 & 7.03 & 1.04 & 1.02 & 0.78 & 0.41 \\
\hline
\end{tabular}

Table 7 demonstrates that weight and size of 100 berries and berry length and width were greatly improved by foliar spraying with glycine at 250,500 , and $750 \mathrm{ppm}$, MLAE at 4 and $6 \%$, the combinations of glycine $500 \mathrm{ppm}+$ FA $100 \mathrm{ppm}+$ MLAE $4 \%$ and glycine $750 \mathrm{ppm}+$ FA $150 \mathrm{ppm}+$ MLAE 6\% compared with that of the control. The highest obvious effect was obtained by the spraying of glycine at 750 followed by $500 \mathrm{ppm}$ comparing with the other applied treatments, in both experimental seasons. 
Table 7. Influence of the Foliar Application of Glycine, FA, MLAE, and Their Combinations on Weight, and Size of 100 Berries, Berry Length, and Width of Grape cv. 'Flame Seedless' During 2018 and 2019 Seasons

\begin{tabular}{|c|c|c|c|c|c|c|c|c|}
\hline \multirow{2}{*}{ Treatment } & \multicolumn{2}{|c|}{$\begin{array}{l}\text { Weight of } 100 \\
\text { Berries }(\mathrm{g})\end{array}$} & \multicolumn{2}{|c|}{$\begin{array}{c}\text { Size of } 100 \text { Berries } \\
\left(\mathrm{cm}^{3}\right)\end{array}$} & \multicolumn{2}{|c|}{$\begin{array}{l}\text { Berry Length } \\
(\mathrm{mm})\end{array}$} & \multicolumn{2}{|c|}{$\begin{array}{l}\text { Berry Width } \\
(\mathrm{mm})\end{array}$} \\
\hline & 2018 & 2019 & 2018 & 2019 & 2018 & 2019 & 2018 & 2019 \\
\hline Control & $196.60 \mathrm{i}$ & $200.00 \mathrm{k}$ & $175.60 \mathrm{j}$ & $178.40 \mathrm{j}$ & $14.94 \mathrm{i}$ & $15.17 \mathrm{~h}$ & $14.57 \mathrm{j}$ & $14.86 \mathrm{j}$ \\
\hline Glycine 250 ppm & $265.2 b c$ & $273.20 c$ & $241.20 c$ & $248.00 c$ & $17.26 \mathrm{c}$ & $17.25 \mathrm{c}$ & $16.27 d$ & $17.29 c$ \\
\hline Glycine 500 ppm & $275.00 \mathrm{a}$ & $287.40 \mathrm{a}$ & $259.00 a$ & $263.40 \mathrm{~b}$ & $17.72 b$ & $17.98 \mathrm{~b}$ & $16.57 \mathrm{c}$ & $17.58 b$ \\
\hline Glycine 750 ppm & $277.00 a$ & $288.20 a$ & $259.40 a$ & $274.60 a$ & $18.00 \mathrm{a}$ & $18.64 a$ & $17.14 \mathrm{a}$ & $18.41 \mathrm{a}$ \\
\hline FA 50 ppm & $208.80 \mathrm{~h}$ & $212.00 \mathrm{j}$ & $188.20 \mathrm{i}$ & $189.60 \mathrm{i}$ & $16.17 f$ & $16.71 \mathrm{e}$ & $15.43 \mathrm{~g}$ & $15.69 \mathrm{~g}$ \\
\hline FA 100 ppm & $215.00 \mathrm{~g}$ & $220.00 \mathrm{i}$ & 193.60h & $197.80 \mathrm{~h}$ & $15.43 \mathrm{~h}$ & $16.05 \mathrm{~g}$ & $14.87 i$ & $15.15 i$ \\
\hline FA 150 ppm & $219.00 f$ & $226.20 \mathrm{~h}$ & $200.00 \mathrm{~g}$ & $202.40 \mathrm{~g}$ & $15.87 \mathrm{~g}$ & $16.39 f$ & $15.21 \mathrm{~h}$ & $15.41 \mathrm{~h}$ \\
\hline MLAE $2 \%$ & $244.40 \mathrm{e}$ & $247.60 \mathrm{~g}$ & $221.20 f$ & $229.00 f$ & $15.50 \mathrm{~h}$ & $16.15 \mathrm{~g}$ & $14.97 i$ & $15.86 \mathrm{~g}$ \\
\hline MLAE 4\% & $247.20 \mathrm{e}$ & $259.00 \mathrm{e}$ & $234.20 d$ & $239.60 d$ & $16.41 \mathrm{e}$ & $17.22 \mathrm{c}$ & $15.71 f$ & $16.52 \mathrm{e}$ \\
\hline MLAE $6 \%$ & $259.40 d$ & $268.00 d$ & $242.20 c$ & $247.80 c$ & $16.78 d$ & $17.95 b$ & $16.20 d$ & $16.62 \mathrm{e}$ \\
\hline $\begin{array}{l}\text { Glycine } 250 \text { ppm } \\
+ \text { FA } 50 \text { ppm + } \\
\text { MLAE } 2 \%\end{array}$ & $244.60 \mathrm{e}$ & $251.40 f$ & $226.20 \mathrm{e}$ & $233.60 \mathrm{e}$ & $16.07 f$ & $16.93 d$ & $15.25 h$ & 16.10f \\
\hline $\begin{array}{l}\text { Glycine } 500 \mathrm{ppm} \\
+ \text { FA } 100 \mathrm{ppm}+ \\
\text { MLAE } 4 \%\end{array}$ & $\begin{array}{l}262.40 c \\
d\end{array}$ & $272.00 \mathrm{c}$ & $242.00 c$ & $251.40 c$ & $17.20 c$ & $17.77 b$ & $16.05 \mathrm{e}$ & $16.97 d$ \\
\hline $\begin{array}{l}\text { Glycine } 750 \text { ppm } \\
\text { + FA } 150 \text { ppm + } \\
\text { MLAE } 6 \%\end{array}$ & $266.00 \mathrm{~b}$ & 279.00b & $251.4 b$ & $259.40 \mathrm{~b}$ & $16.71 d$ & $18.56 a$ & $16.74 b$ & $17.49 b$ \\
\hline LSD 0.05 & 3.33 & 3.25 & 4.81 & 4.18 & 0.16 & 0.22 & 0.11 & 0.17 \\
\hline
\end{tabular}

Table 8 demonstrates that the foliar spraying with glycine at 250, 500, and $750 \mathrm{ppm}$ and with MLAE at 4 and $6 \%$ produced increases in the juice percentage and anthocyanin concentration as compared with the control in both seasons. In addition, these parameters were significantly enhanced by spraying with glycine $500 \mathrm{ppm}+$ FA $100 \mathrm{ppm}+$ MLAE $4 \%$ and with glycine $750 \mathrm{ppm}+\mathrm{FA} 150 \mathrm{ppm}+$ MLAE $6 \%$ compared with the other applied treatments and the control in the two experimental seasons. The percentages of TSS and total sugars were remarkably improved by foliar spraying with glycine at 250,500 , and $750 \mathrm{ppm}$, with MLAE at 2, 4 and 6\%, and by the combinations of glycine $500 \mathrm{ppm}+\mathrm{FA}$ $100+$ MLAE $4 \%$ and glycine $750 \mathrm{ppm}+$ FA $150 \mathrm{ppm}+$ MLAE 6\%. However, these treatments decreased the percentage of fruit acidity in both seasons compared with the other applied treatments and control. 
Table 8. Influence of the Foliar Application of Glycine, FA, MLAE, and Their Combinations on the Percentages of Juice, Anthocyanin, TSS, Total Sugar, and Total Acidity in Fruit of Grape cv. 'Flame Seedless' During 2018 and 2019 Seasons

\begin{tabular}{|c|c|c|c|c|c|c|c|c|c|c|}
\hline \multirow{2}{*}{ Treatment } & \multicolumn{2}{|c|}{ Juice (\%) } & \multicolumn{2}{|c|}{$\begin{array}{l}\text { Anthocyanin } \\
(\mathrm{mg} / 100 \mathrm{~g})\end{array}$} & \multicolumn{2}{|c|}{ TSS (\%) } & \multicolumn{2}{|c|}{ Total Sugar (\%) } & \multicolumn{2}{|c|}{ Total Acidity (\%) } \\
\hline & 2018 & 2019 & 2018 & 2019 & 2018 & 2019 & 2018 & 2019 & 2018 & 2019 \\
\hline Control & $138.80 \mathrm{i}$ & $142.20 f$ & $0.28 \mathrm{f}$ & $0.28 \mathrm{~g}$ & $16.50 \mathrm{~h}$ & $18.00 \mathrm{~d}$ & $13.78 \mathrm{~g}$ & $15.00 \mathrm{~d}$ & $0.73 a$ & $0.74 a$ \\
\hline Glycine 250 ppm & 197.00b & $210.20 b$ & $0.55 b c$ & $0.58 \mathrm{bc}$ & 19.03bcd & $19.74 a b c$ & $16.16 \mathrm{bcd}$ & $16.09 \mathrm{~cd}$ & $0.45 \mathrm{e}$ & $0.46 \mathrm{e}$ \\
\hline Glycine 500 ppm & $212.40 a$ & $218.00 \mathrm{a}$ & $0.56 \mathrm{~b}$ & $0.59 b$ & $19.50 a b c$ & $20.09 a b$ & $16.84 \mathrm{bc}$ & $16.77 \mathrm{bc}$ & $0.35 f g$ & $0.38 f$ \\
\hline Glycine 750 ppm & $214.80 a$ & $224.80 \mathrm{a}$ & $0.67 a$ & $0.69 a$ & $19.97 a b$ & $20.68 a$ & 17.29ab & $17.46 \mathrm{~b}$ & $0.32 \mathrm{~g}$ & $0.33 \mathrm{~g}$ \\
\hline FA $50 \mathrm{ppm}$ & $148.00 \mathrm{~h}$ & $157.00 \mathrm{e}$ & $0.34 f$ & $0.47 e$ & $16.90 \mathrm{gh}$ & $18.10 \mathrm{~d}$ & $14.28 \mathrm{fg}$ & $15.04 d$ & $0.65 b$ & $0.63 b$ \\
\hline FA $100 \mathrm{ppm}$ & 148.60h & $169.00 d$ & $0.44 \mathrm{e}$ & $0.51 \mathrm{de}$ & $17.50 \mathrm{fgh}$ & $18.60 \mathrm{~cd}$ & $14.68 \mathrm{efg}$ & $15.18 d$ & $0.57 c$ & $0.58 \mathrm{c}$ \\
\hline FA $150 \mathrm{ppm}$ & $160.00 . \mathrm{g}$ & $169.80 \mathrm{~cd}$ & $0.44 \mathrm{e}$ & $0.51 \mathrm{de}$ & $17.53 \mathrm{fgh}$ & $18.87 \mathrm{bcd}$ & 14.96efg & $15.44 d$ & $0.53 d$ & $0.56 \mathrm{~cd}$ \\
\hline MLAE 2\% & $164.20 \mathrm{fg}$ & $175.80 \mathrm{~cd}$ & $0.43 \mathrm{e}$ & $0.41 f$ & 17.90efg & $18.90 \mathrm{bcd}$ & 15.34def & $15.56 \mathrm{~d}$ & $0.55 \mathrm{~cd}$ & $0.56 \mathrm{~cd}$ \\
\hline MLAE 4\% & 171.40de & $178.20 \mathrm{~cd}$ & $0.48 \mathrm{cde}$ & $0.54 \mathrm{bcd}$ & 18.20def & 19.47abc & $15.73 \mathrm{cde}$ & $16.82 \mathrm{bc}$ & $0.54 \mathrm{~cd}$ & $0.53 d$ \\
\hline MLAE 6\% & $176.60 \mathrm{~d}$ & $180.20 \mathrm{c}$ & $0.48 \mathrm{cde}$ & $0.55 \mathrm{bcd}$ & 18.83cde & $19.68 \mathrm{abc}$ & $16.26 \mathrm{bcd}$ & $17.47 \mathrm{~b}$ & $0.46 \mathrm{e}$ & $0.47 \mathrm{e}$ \\
\hline $\begin{array}{l}\text { Glycine } 250 \text { ppm + FA } 50 \text { ppm + } \\
\text { MLAE } 2 \%\end{array}$ & 167.00 ef & $170.40 \mathrm{~cd}$ & $0.46 \mathrm{de}$ & $0.53 \mathrm{cde}$ & 18.06def & 19.10bcd & 15.37def & $16.07 \mathrm{~cd}$ & $0.54 \mathrm{~cd}$ & $0.55 \mathrm{~cd}$ \\
\hline $\begin{array}{l}\text { Glycine } 500 \text { ppm + FA } 100 \text { ppm } \\
\text { + MLAE } 4 \%\end{array}$ & $186.60 \mathrm{c}$ & $193.60 \mathrm{~b}$ & $0.5 \mathrm{bcde}$ & $0.55 \mathrm{bcd}$ & $19.73 a b c$ & $20.07 a b$ & $16.92 b$ & $17.83 b$ & $0.43 e$ & $0.44 \mathrm{e}$ \\
\hline $\begin{array}{l}\text { Glycine } 750 \text { ppm + FA } 150 \text { ppm } \\
\text { + MLAE } 6 \%\end{array}$ & $189.20 \mathrm{c}$ & $194.60 \mathrm{~b}$ & $0.52 \mathrm{bcd}$ & $0.55 \mathrm{bcd}$ & $20.23 a$ & $20.62 a$ & $18.14 a$ & $19.00 \mathrm{a}$ & $0.36 f$ & $0.35 \mathrm{fg}$ \\
\hline LSD 0.05 & 6.70 & 10.41 & 0.08 & 0.057 & 1.03 & 1.28 & 1.18 & 1.09 & 0.03 & 0.04 \\
\hline
\end{tabular}




\section{Leaf Chemical Composition}

Table 9 shows that the leaf composition of N, P, and K, was remarkably increased by spraying glycine at 500 and $750 \mathrm{ppm}$, MLAE at 4 and 6\% comparing with control or the rest treatments in the two seasons. In addition, the highest leaf content from $\mathrm{N}, \mathrm{P}$, and $\mathrm{K}$ was correlated by spraying the combinations of glycine $500 \mathrm{ppm}+\mathrm{FA} 100 \mathrm{ppm}+\mathrm{MLAE}$ $4 \%$ and glycine $750 \mathrm{ppm}+\mathrm{FA} 150 \mathrm{ppm}+$ MLAE $6 \%$.

Table 9. Influence of the Foliar Application of Glycine, FA, MLAE, and Their Combinations on Leaf content from N, P, and K of Grape cv. 'Flame Seedless' during 2018 and 2019 Seasons

\begin{tabular}{|c|c|c|c|c|c|c|}
\hline \multirow{2}{*}{ Treatment } & \multicolumn{2}{|c|}{$\mathrm{N} \%$} & \multicolumn{2}{|c|}{$\mathrm{P} \%$} & \multicolumn{2}{|c|}{$\mathrm{K} \%$} \\
\hline & 2018 & 2019 & 2018 & 2019 & 2018 & 2019 \\
\hline Control & $0.22 \mathrm{~h}$ & $0.34 \mathrm{def}$ & $0.28 \mathrm{hi}$ & $0.31 \mathrm{~h}$ & $4.14 \mathrm{e}$ & $3.98 \mathrm{i}$ \\
\hline Glycine 250 ppm & $0.35 \mathrm{ef}$ & $0.38 \mathrm{bcde}$ & $0.42 \mathrm{e}$ & $0.44 \mathrm{e}$ & $5.56 c$ & $5.80 \mathrm{~cd}$ \\
\hline Glycine 500 ppm & $0.43 \mathrm{bcd}$ & $0.41 b$ & $0.49 d$ & $0.52 d$ & $5.88 \mathrm{abc}$ & $6.30 \mathrm{~b}$ \\
\hline Glycine 750 ppm & $0.45 b c$ & $0.50 \mathrm{a}$ & $0.59 c$ & $0.62 \mathrm{c}$ & $5.88 \mathrm{abc}$ & $6.31 \mathrm{~b}$ \\
\hline FA 50 ppm & $0.26 \mathrm{gh}$ & $0.29 f$ & $0.22 \mathrm{j}$ & $0.24 \mathrm{i}$ & $3.90 \mathrm{e}$ & $3.84 \mathrm{i}$ \\
\hline FA $100 \mathrm{ppm}$ & $0.32 \mathrm{fg}$ & 0.33 ef & $0.24 \mathrm{ij}$ & $0.24 \mathrm{i}$ & $4.08 \mathrm{e}$ & $4.42 \mathrm{~h}$ \\
\hline FA $150 \mathrm{ppm}$ & $0.36 \mathrm{ef}$ & $0.35 \mathrm{cde}$ & $0.27 \mathrm{hi}$ & $0.26 \mathrm{i}$ & $4.72 \mathrm{~d}$ & $5.04 \mathrm{~g}$ \\
\hline MLAE $2 \%$ & $0.27 \mathrm{gh}$ & $0.38 \mathrm{bcde}$ & $0.29 \mathrm{~h}$ & $0.31 \mathrm{~h}$ & $4.86 \mathrm{~d}$ & 5.40ef \\
\hline MLAE 4\% & 0.37 def & $0.39 \mathrm{bcd}$ & $0.34 \mathrm{~g}$ & $0.37 \mathrm{~g}$ & $5.60 \mathrm{bc}$ & $5.18 \mathrm{fg}$ \\
\hline MLAE 6\% & $0.40 \mathrm{cde}$ & $0.41 b c$ & $0.40 \mathrm{ef}$ & $0.40 \mathrm{fg}$ & $5.72 \mathrm{bc}$ & $6.00 c$ \\
\hline $\begin{array}{l}\text { Glycine } 250 \text { ppm + FA } 50 \text { ppm + } \\
\text { MLAE } 2 \%\end{array}$ & $0.39 \mathrm{cde}$ & $0.40 \mathrm{bc}$ & $0.37 f$ & 0.42ef & $4.92 d$ & $5.60 \mathrm{de}$ \\
\hline $\begin{array}{l}\text { Glycine } 500 \text { ppm + FA } 100 \text { ppm + } \\
\text { MLAE } 4 \%\end{array}$ & $0.47 a b$ & $0.52 a$ & $0.83 b$ & $0.88 b$ & $5.92 \mathrm{ab}$ & $6.46 \mathrm{~b}$ \\
\hline $\begin{array}{l}\text { Glycine } 750 \text { ppm + FA } 150 \text { ppm + } \\
\text { MLAE } 6 \%\end{array}$ & $0.52 \mathrm{a}$ & $0.55 a$ & $0.91 a$ & $0.91 a$ & $6.16 a$ & $7.24 a$ \\
\hline LSD 0.05 & 0.06 & 0.05 & 0.03 & 0.03 & 0.34 & 0.26 \\
\hline
\end{tabular}

The data obtained shows that the foliar spraying with glycine at 250, 500, and 750 ppm had an effective role in improving the vegetative growth parameters, yield, and fruit quality characteristics of 'Flame Seedless' grape cultivar. These results were previously explained by Sekhon (2003) and Souri (2016). They reported that glycine amino acid is the smallest amino acid and can produce chelates for different ions of elements and products. The stimulatory effect of glycine amino acid increases under the conditions of stress such as salinity and water stress (Rai 2002; Cerdán et al. 2013; Sadak et al. 2015; Souri et al. 2018). Besides, the application of glycine increased TSS and vitamin C compared with the control (Sekhon 2003; Liu et al. 2011; Shan et al. 2016; Razavi et al. 2018; Mohammadipour and Souri 2019). Moreover, they found that leaf nutrient concentrations of $\mathrm{N}, \mathrm{Ca}, \mathrm{K}, \mathrm{P}, \mathrm{Mg}, \mathrm{Mn}, \mathrm{Fe}$, and $\mathrm{Zn}$, were significantly increased by soil application of glycine compared with the unfertilized control plants. Glycine amino acids can play a crucial role in raising the stability of cell membrane and its protection from peroxidation through the growth of plant under the stresses conditions (Keutgen and Pawelzik 2008; Zobiole et al. 2012; Rizwan et al. 2017; Teixeira et al. 2017). It was mentioned by many authors that the transfer of nutrients in tissues of plants is related greatly to the glycine concentration and status (Marschner 2011; Souri 2016). In addition, glycine has a crucial role in the total chlorophyll, forming vegetative growth, and in increasing the availability of $\mathrm{Fe}, \mathrm{Zn}, \mathrm{Mn}$, and $\mathrm{Cu}$ to plants (Ghasemi et al. 2013). The application of nitrogen reduced 
compounds such glycine amino acid raised the chlorophyll content in the leaves (Fahimi et al. 2016; Souri et al. 2017). Parameters of coriander (Coriandrum sativum) plant growth, plant height, leaf SPAD value, and shoot and root fresh weights were significantly improved by applying glycine, particularly at high concentrations (Souri and Hatamian 2019). Additionally, the same authors reported that, because of the simple process for glycine synthesis, it could be combined with the nutrients producing chelates to increase the absorption of the elements and transfer them into the plants. Spraying glycine amino acids on apple trees (Malus domestica L. Borkh) cv. 'Anna' at 25, 50, and $100 \mathrm{ppm}$ improved shoot length, shoot diameter, total chlorophyll, yield, fruit weight, size, length, diameter, TSS, total sugars, anthocyanin, and leaf composition from macro and micronutrients, while it reduced the total acidity and fruit drop percentage. Moreover, they noticed that the effect of 50 and $100 \mathrm{ppm}$ was higher than $25 \mathrm{ppm}$ (Mosa et al. 2021).

The results of this study show that the foliar spraying of folic acid (FA) had a good effect on the vegetative growth parameters, yield, and fruit quality of grape cv. 'Flame Seedless', especially when it was applied at $150 \mathrm{ppm}$. These results were in harmony with the previous findings of Stakhova et al. (2000). They stated that foliar spraying of FA enhanced the photosynthetic rate in the leaves, seed weight, and yield of peas (Pisum sativum L.). FA at $50 \mu \mathrm{m}$ raised the yield, leaf mineral content, seed weight, and quality of the seed and leaf chlorophyll rate (Burguieres et al. 2007). Moreover, Kim (2007) demonstrated that folate plays an important role in the synthesis and repetition of DNA by arranging the transporting carbon unites, which participates in purines and thymidylate synthesis. In the same trend, Fardet et al. (2008) found that FA has a positive influence in increasing the vegetative growth, yield, and fruit quality in many plant species because they can catch the free radicals and active oxygen, which are produced during the processes of photosynthesis and respiration. It was noticed by many authors that folates are an important factor in helping the transferring of carbon as donors and acceptors, which can engage in purines, pyrimidines, and amino acids synthesis (Dhonukshe-Rutten et al. 2009; Blancquaert et al. 2010). Treating flax plants with FA greatly increases the parameters of growth, photosynthetic rate, number of flowers, maturity index, yield, and quality of fiber and seeds (Emam et al. 2011). In Pisum sativum L. cv. 'Master-B', Farouk and Abdul Qados (2018) found that spraying FA at $20 \mathrm{mg} / \mathrm{L}$ significantly increased the height of plant, weight of shoot and dry shoots, area of plant leaf, pigments of photosynthesis, yield, and fruit quality.

The results show that MLAE plays an important role in improving the vegetative growth, yield, fruit quality, and leaf composition from $\mathrm{N}, \mathrm{P}$, and $\mathrm{K}(\%)$ compared with the control. These results are in parallel with the findings of other studies of (Dillard and German 2000; Siddhuraju and Becker 2003; Aslam et al. 2005). They mentioned that moringa leaf extracts have good quantities from $\beta$-carotene, protein, vitamin $\mathrm{C}, \mathrm{Ca}, \mathrm{K} \mathrm{Mg}$, $\mathrm{K}, \mathrm{Mn}, \mathrm{P}, \mathrm{Zn}, \mathrm{Na}, \mathrm{Cu}$, and $\mathrm{Fe}$, as well as natural antioxidants such as ascorbic acid, quercetin, zeatin, kaempferol, $\beta$-sitosterol, caffeoylquinic acid, and carotenoids. MLAE increases the content of proline, malondialdehyde, total soluble proteins, and total chlorophyll in spinach (Spinacia oleracea) leaves (Aslam et al. 2005) and yield, fruit quality, juice percentage, and TSS (\%) while it decreases the value of nitrite, including nitrate in the fruit juice of orange trees (Citrus sinensis) (El-Enien et al. 2015). Additionally, Ndong et al. (2007) found that the leaves are a potent source of polyphenols, including quercetin-3-glycoside, rutin, kaempferol, and glycosides. MLAE has increased the growth and yield of a range of plant species such as cereals (Phiri 2010), maize (Basra et al. 2011), and wheat (Sarmin 2014). Culver et al. (2012) reported that MLAE contains relatively high concentrations of the cytokinin, Zeatin so, tomato leaves' fresh and dry weights were enhanced significantly when MLAE is applied at high rates. Spraying MLAE 
stimulated the leaf mineral contents from N, P, and K, leaf area, chlorophyll, shoot length and shoot diameter, yield, as well as physical and chemical characteristics of fruits of Le Conte pear (Abd El-Hamied and El-Amary 2015). Furthermore, MLAE significantly enhanced vegetative growth parameters, total leaf chlorophyll content, and the leaf mineral composition of N, P, and K of pomegranate cv. 'Manfalouti' (Kamel 2015) and grape cv. 'Flame Seedless' (Vitis vinifera L.) (Bassiony and Ibrahim 2016), as well as fruit quality, color, soluble solids content, vitamin $\mathrm{C}$, anthocyanin content, and antioxidant activity of plum cv. 'Hollywood' (Mahmoud et al. 2017). Leone et al. (2015) stated that phenolic acids, carotenoids vitamins, polyphenols, flavonoids, isothiocyanates, alkaloids, glucosinolates, tannins, and saponins exist in moringa leaves with high levels. MLAE has been demonstrated to enhance the production of ornamental and medicinal plants as it was used as an agent for biostimulation. Because of the high micro and macronutrient elements of MLAE, it has a simulative effect on plant growth and plays an important role in photosynthesis and carbohydrate synthesis, which are metabolic processes (Sakr et al. 2018). This might be due to the significant contribution of nitrogen present in MLAE, which caused cell division, cell enlargement, and the overall plant growth (Kanchani and Harris 2019). Mohamed et al. (2020) reported that the application of MLAE on Origanum majorana improved its vegetative growth characteristics and the production of oil.

From the above-discussed results, it could be noticed that the foliar spraying of glycine played an essential role in improving attributes of vegetative growth, yield and fruit quality of grape cv. 'Flame Seedless' compared to the control or the rest of the treatments in the two experimental seasons. The positive effect of glycine was increased in parallel by increasing its concentration, where 750 ppm gave better results rather than 250 or 500 ppm during the two seasons of the study.

\section{CONCLUSIONS}

1. Shoot length, thickness, and leaf total chlorophyll were significantly increased by foliar spraying with glycine at 500 and $750 \mathrm{ppm}$ and with the combination of glycine 750 ppm + FA $150 \mathrm{ppm}+$ MLAE 6\%, which gave the highest increments in the two seasons compared with the control and the other applied treatments.

2. Spraying glycine with 500 and $750 \mathrm{ppm}$ were the best treatments in enhancing the clusters number and weight $(\mathrm{kg})$, thus giving the best yield in $\mathrm{kg}$ per vine or in ton per hectare when compared with the control or the other applied treatments.

3. The best results for TSS (\%), total sugars, juice (\%), and anthocyanin were obtained by the foliar application of glycine at 500 and $750 \mathrm{ppm}$ and by the combination of glycine $750 \mathrm{ppm}+\mathrm{FA} 150 \mathrm{ppm}+$ MLAE $6 \%$ as well as glycine $500 \mathrm{ppm}+\mathrm{FA} 100 \mathrm{ppm}+$ MLAE 4\% when compared with the control and the other applied treatments.

4. The leaf chemical composition of $\mathrm{N}, \mathrm{P}$, and $\mathrm{K}$ was greatly increased by the foliar spray of glycine $750 \mathrm{ppm}+$ FA $150 \mathrm{ppm}+$ MLAE 6\%, which was the superior combination compared with the control and the other applied treatments.

5. In general, the best results were obtained by the foliar spraying of glycine at $750 \mathrm{ppm}$ followed by 500 ppm over all the applied treatments and the control. 


\section{ACKNOWLEDGMENTS}

The authors are grateful to the Deanship of Scientific Research, King Saud University, for funding through the Vice Deanship of Scientific Research Chairs.

\section{REFERENCES CITED}

Abbassy, M. M. S., Salem, M. Z. M., Rashad, N. M., Afify, S. M., and Salem, A. Z. M. (2020). "Nutritive and biocidal properties of agroforestry trees of Moringa oleifera Lam., Cassia fistula L., and Ceratonia siliqua L. as non-conventional edible vegetable oils," A groforestry System 94, 1567-1579. DOI: 10.1007/s10457-018-0325-4

Abd El-Hamied, S. A., and El-Amary, E. I. (2015). "Improving growth and productivity of "pear" trees using some natural plants extracts under north Sinai conditions," IOSR Journal of Agriculture and Veterinary Science. DOI: 10.9790/2380-08110109

Alhakmani, F., Kumar, S., and Khan, S. A. (2013). "Estimation of total phenolic content, in-vitro antioxidant and anti-inflammatory activity of flowers of Moringa oleifera," Asian Pacific Journal of Tropical Biomedicine 3(8), 623-627. DOI: 10.1016/S22211691(13)60126-4

Al-Huqail, A. A., Behiry, S. I., Salem, M. Z. M., Ali, H. M., Siddiqui, M. H., and Salem, A. Z. M. (2019). "Antifungal, antibacterial, and antioxidant activities of Acacia saligna (Labill.) H. L. Wendl. flower extract: HPLC analysis of phenolic and flavonoid compounds," Molecules 24 (4), Article Number 700. DOI: 10.3390/molecules 24040700

Al-Maliky, A. W., Jerry, A. N., and Obead, F. T. (2019). "The effect of foliar spraying with folic acid and cysteine on growth and yield of green bean plants (Vicia faba L.)," Journal of Al-Muthanna for Agricultural Sciences 7(4), 22-28. DOI: 10.18081/MJAS/2019-7/22-28

Arrobas, M., Afonso, S., and Rodrigues, M. Â. (2018). "Diagnosing the nutritional condition of chestnut groves by soil and leaf analyses," Scientia Horticulturae 228, 113-121. DOI: 10.1016/j.scienta.2017.10.027

Ashmawy, N. A., Salem, M. Z. M., El Shanhorey, N., Al-Huqail, A. A., Ali, H. M., and Behiry, S. I. (2020). "Eco-friendly wood-biofungicidal and antibacterial activities of various Coccoloba uvifera L. leaf extracts: HPLC analysis of phenolic and flavonoid compounds," BioResources 15(2), 4165-4187. DOI: 10.15376/biores.15.2.4165-4187

Aslam, M., Anwar, F., Nadeem, R., Rashid, U., Kazi, T. G., and Nadeem, M. (2005). "Mineral composition of Moringa oleifera leaves and pods from different regions of Punjab, Pakistan," Asian Journal of Plant Sciences 4(4), 417-421. DOI: 10.3923/ajps.2005.417.421

Banerjee, P., and Prasad, B. (2020). "Determination of concentration of total sodium and potassium in surface and ground water using a flame photometer," Applied Water Science 10, Article Number 113. DOI: 10.1007/s13201-020-01188-1

Basra, S. M. A., Iftikhar, M. N., and Afzal, I. (2011). "Potential of moringa (Moringa oleifera) leaf extract as priming agent for hybrid maize seeds," International Journal of Agriculture and Biology 13(6), 1006-1010.

Bassiony, S. S., and Ibrahim, M. G. (2016). "Effect of silicon foliar sprays combined with Moringa leaves extract on yield and fruit quality of 'Flame Seedless' grape (Vitis 
vinifera L.)," Journal of Plant Production 7(10), 1127-1135. DOI:

10.21608/jpp.2016.46946

Behiry, S. I., Okla, M. K., Alamri, S. A., El-Hefny, M., Salem, M. Z. M., Alaraidh, I. A., Ali, H. M., Al-Ghtani, S. M., Monroy, J. C., and Salem, A. Z. M. (2019). “Antifungal and antibacterial activities of Musa paradisiaca L. peel extract: HPLC analysis of phenolic and flavonoid contents," Processes 7(4), Article Number 215. DOI: 10.3390/pr7040215

Blancquaert, D., Storozhenko, S., Loizeau, K., De Steur, H., De Brouwer, V., Viaene, J., and van der Straeten, D. (2010). "Folates and folic acid: From fundamental research toward sustainable health," Critical Reviews in Plant Science 29(1), 14-35. DOI: 10.1080/07352680903436283

Burguieres, E., McCue, P., Kwon, Y.-I., and Shetty, K. (2007). "Effect of vitamin C and folic acid on seed vigour response and phenolic-linked antioxidant activity," Bioresource Technology 98(7), 1393-1404. DOI: 10.1016/j.biortech.2006.05.046

Carr, A. C. and Maggini, S. (2017). "Vitamin C and immune function," Nutrients 9(11), 1211. DOI: $10.3390 /$ nu9111211

Cerdán, M., Sánchez-Sánchez, A., Jordá, J. D., Juárez, M., and Sánchez-Andreu, J. (2013). "Effect of commercial amino acids on iron nutrition of tomato plants grown under lime-induced iron deficiency," Journal of Plant Nutrition and Soil Science 176(6), 859-866. DOI: 10.1002/jpln.201200525

Culver, M., Fanuel, T., and Chiteka, A. Z. (2012). "Effect of moringa extract on growth and yield of tomato," Greener Journal of Agricultural Sciences 2(5), 207-211. DOI: 10.15580/GJAS.2012.5.GJAS1233

Dhonukshe-Rutten, R. A. M., de Vries, J. H. M., de Bree, A., van der Put, N., Van Staveren, W. A., and de Groot, L. C. P. G. M. (2009). "Dietary intake and status of folate and vitamin B12 and their association with homocysteine and cardiovascular disease in European populations," European Journal of Clinical Nutrition 63(1), 18 30. DOI: $10.1038 /$ sj.ejcn.1602897

Dillard, C. J., and German, J. B. (2000). "Phytochemicals: Nutraceuticals and human health," Journal of the Science of Food and Agriculture 80(12), 1744-1756. DOI: 10.1002/1097-0010(20000915)80:12<1744: AID-JSFA725>3.0.CO; 2-W

Drosou, C., Kyriakopoulou, K., Bimpilas, A., Tsimogiannis, D., and Krokida, M. (2015). "A comparative study on different extraction techniques to recover red grape pomace polyphenols from vinification byproducts," Industrial Crops and Products 75, 141149. DOI: 10.1016/j.indcrop.2015.05.063

El-Enien, M. S. A., El-Azazy, A. M., and El-Sayed, F. S. (2015). "Effect of moringa leaves extract as a natural product compared with other synthetic compounds on yield production and fruit quality of navel orange trees," Egyptian Journal of Horticulture 42(2), 899-911. DOI: 10.21608/ejoh.2015.1339

Emam, M. M., El-Sweify, A. H., and Helal, N. M. (2011). "Efficiencies of some vitamins in improving yield and quality of flax plant," African Journal of Agricultural Research 6(18), 4362-4369. DOI: 10.5897/AJAR11.1104

Fahimi, F., Souri, M. K., and Yaghobi, F. (2016). "Growth and development of greenhouse cucumber under foliar application of Biomin and Humifolin fertilizers in comparison to their soil application and NPK," Journal of Science and Technology of Greenhouse Culture 7(1), 143-152. DOI: 10.18869/acadpub.ejgcst.7.1.143 
Fardet, A., Rock, E., and Rémésy, C. (2008). "Is the in vitro antioxidant potential of whole-grain cereals and cereal products well reflected in vivo?," Journal of Cereal Science 48(2), 258-276. DOI: 10.1016/j.jcs.2008.01.002

Farouk, S., and Qados, A. M. S. A. (2018). "Enhancing seed quality and productivity as well as physio-anatomical responses of pea plants by folic acid and/or hydrogen peroxide application," Scientia Horticulturae 240, 29-37. DOI:

10.1016/j.scienta.2018.05.049

Ghasemi, S., Khoshgoftarmanesh, A. H., Hadadzadeh, H., and Afyuni, M. (2013). "Synthesis, characterization, and theoretical and experimental investigations of zinc(II)-amino acid complexes as ecofriendly plant growth promoters and highly bioavailable sources of zinc," Journal of Plant Growth Regulation 32(2), 315-323. DOI: $10.1007 / \mathrm{s} 00344-012-9300-\mathrm{x}$

Gibson, P. R., and Shepherd, S. J. (2012). "Food choice as a key management strategy for functional gastrointestinal symptoms," The American Journal of Gastroenterology 107(5), 657-666. DOI:10.1038/ajg.2012.49.

Gibson, P. R. (2017). "Use of the low-FODMAP diet in inflammatory bowel disease," Journal of Gastroenterology and Hepatology 32(1), 40-42. DOI:10.1111/jgh.13695.

Hogan, S., Zhang, L., Li, J., Sun, S., Canning, C., and Zhou, K. (2010). “Antioxidant rich grape pomace extract suppresses postprandial hyperglycemia in diabetic mice by specifically inhibiting alpha-glucosidase," Nutrition \& Metabolism 7(1), 1-9. DOI: 10.1186/1743-7075-7-71

Ibrahim, M. F. M., Ibrahim, H. A., and Abd El-Gawad, H. G. (2021). "Folic acid as a protective agent in snap bean plants under water deficit conditions," The Journal of Horticultural Science and Biotechnology 96(1), 94-109. DOI: 10.1080/14620316.2020.1793691

Iqbal, S., and Bhanger, M. I. (2006). "Effect of season and production location on antioxidant activity of Moringa oleifera leaves grown in Pakistan," Journal of Food Composition and Analysis 19(6-7), 544-551. DOI: 10.1016/j.jfca.2005.05.001

Iriti, M., and Faoro, F. (2006). "Grape phytochemicals: A bouquet of old and new nutraceuticals for human health," Medical Hypotheses 67, 833-838. DOI: 10.1016/j.mehy.2006.03.049

Jiang, J., Fan, X., Zhang, Y., Tang, X., Li, X., Liu, C., and Zhang, Z. (2020). "Construction of a high-density genetic map and mapping of firmness in grapes (Vitis vinifera L.) based on whole-genome resequencing," International Journal of Molecular Sciences 21(3), Article Number 797. DOI: 10.3390/ijms21030797

Kamel, H. M. (2015). "Response of Manfalouty pomegranate transplants to foliar spray and soil drench applications with some natural extracts," Journal of Horticultural Science \& Ornamental Plants 7(3), 107-116. DOI: 10.13140/RG.2.1.3875.5928

Kanchani, A. M. K. D. M., and Harris, K. D. (2019). "Effect of foliar application of moringa (Moringa oleifera) leaf extract with recommended fertilizer on growth and yield of okra (Abelmoschus esculentus)," AGRIEAST: Journal of Agricultural Sciences 13(2), 38-54. DOI: 10.4038/agrieast.v13i2.73

Keutgen, A., and Pawelzik, E. (2008). "Contribution of amino acids to strawberry fruit quality and their relevance as stress indicators under $\mathrm{NaCl}$ salinity," Food Chemistery 111(3), 642-647. DOI: 10.1016/j.foodchem.2008.04.032.

Kim, Y. I. (2007). "Folate and colorectal cancer: An evidence-based critical review," Molecular Nutrition \& Food Research 51(3), 267-92. DOI: 10.1002/mnfr.200600191 
Leone, A., Spada, A., Battezzati, A., Schiraldi, A., Aristil, J., and Bertoli, S. (2015). "Cultivation, genetic, ethnopharmacology, phytochemistry and pharmacology of Moringa oleifera leaves: An overview," International Journal of Molecular Sciences 16(6), 12791-12835. DOI: 10.3390/ijms 160612791

Li, D., Li, L., Luo, Z., Mou, W., Mao, L., and Ying, T. (2015). “Comparative transcriptome analysis reveals the influence of abscisic acid on the metabolism of pigments, ascorbic acid and folic acid during strawberry fruit ripening," PLOS ONE 10(6), e0130037. DOI: 10.1371/journal.pone.0130037

Liu, J., Wisniewski, M., Droby, S., Vero, S., Tian, S., and Hershkovitz, V. (2011). "Glycine betaine improves oxidative stress tolerance and biocontrol efficacy of the antagonistic yeast Cystofilobasidium infirmominiatum," International Journal of Food Microbiology 146(1), 76-83. DOI: 10.1016/j.ijfoodmicro.2011.02.007

Lo'ay, A. A., and Doaa, M. H. (2020). "The potential of vine rootstocks impacts on 'Flame Seedless' bunches behavior under cold storage and antioxidant enzyme activity performance," Scientia Horticulturae 260, Article ID 108844. DOI: 10.1016/j.scienta.2019.108844

Lund, S., and Bohlmann, J. (2006). "The molecular basis for wine grape quality - A volatile subject," Science 311(5762), 804-805. DOI: 10.1126/science.1118962. PMID: 16469915

Mahmoud, T. Sh. M., Kassim, N. E., AbouRayya, M. S., and Abdalla, A. M. (2017). "Influence of foliar application with moringa (Moringa oleifera L.) leaf extract on yield and fruit quality of Hollywood plum cultivar," Journal of Horticulture 4(193), 1-7. DOI: 10.4172/2376-0354.1000193.

Makkar, H. P. S., Francis, G., and Becker, K. (2007). "Bioactivity of phytochemicals in some lesser-known plants and their effects and potential applications in livestock and aquaculture production systems," Animal 1(9), 1371-1391. DOI: $10.1017 /$ S1751731107000298

Mansour, M. M. (2014). "Response of soybean plants to exogenously applied with ascorbic acid, zinc sulphate and paclobutrazol," Report and Opinion 6(11), 17-25. DOI: $10.7537 /$ marsroj061114.04

Marschner, P. (2011). Marschner's Mineral Nutrition of Higher Plants, $3^{\text {rd }}$ Ed., P. Marschner (ed.), Elsevier/Academic Press Amsterdam, Netherlands, pp. 684. ISBN 978-0-12-384905-2

Mohamed, A. A., El-Hefny, M., El-Shanhorey, N. A., and Ali, H. M. (2020). "Foliar application of bio-stimulants enhancing the production and the toxicity of Origanum majorana essential oils against four rice seed-borne fungi," Molecules 25(10), Article Number 2363. DOI: 10.3390/molecules25102363

Mohammadipour, N., and Souri, M. K. (2019). "Beneficial effects of glycine on growth and leaf nutrient concentrations of coriander (Coriandrum sativum) plants," Journal of Plant Nutrition 42(14), 1637-1644. DOI: 10.1080/01904167.2019.1628985

Monagas, M., Hernandez-Ledesma, B., Gomez-Cordoves, C., and Bartolome, B. (2006). "Commercial dietary ingredients from Vitis vinifera L. leaves and grape skins: Antioxidant and chemical characterization" Journal of Agricultural and Food Chemistry 54, 319-327. DOI: 10.1021/jf051807j.

Moores, J. (2013). “Vitamin, C: A wound healing perspective," British Journal of Community Nursing 6, 8-11. DOI:10.12968/bjen.2013.18.sup12.s6

Mosa, W. F. A., Ali, H. M., and Abdelsalam, N. R. (2021). "The utilization of tryptophan and glycine amino acids as safe alternatives to chemical fertilizers in apple orchards," 
Environmental Science and Pollution Research 28(3), 1983-1991. DOI:

10.1007/s11356-020-10658-7

Nangle, E. J., Gardner, D. S., Metzger, J. D., Rodriguez-Saona, L., Guisti, M. M., Danneberger, T. K., and Petrella, D. P. (2015). "Pigment changes in cool-season turfgrasses in response to ultraviolet-B light irradiance," Agronomy Journal 107(1), 41-50. DOI: 10.2134/agronj14.0249

Ndong, M., Uehara, M., Katsumata, S.-I., and Suzuki, K. (2007). "Effects of oral administration of Moringa oleifera Lam on glucose tolerance in Goto-Kakizaki and Wistar rats," Journal of Clinical Biochemistry and Nutrition 40(3), 229-233. DOI: $10.3164 /$ jcbn.40.229

Nielsen, S. S. (2010). "Phenol-sulfuric acid method for total carbohydrates," in: Food Analysis Laboratory Manual, Food Science Texts Series, S. S. Nielsen (ed.), Springer, Boston, MA, USA, pp. 47-53.

Ott, R. L., and Longnecker, M. T. (2015). An Introduction to Statistical Methods and Data Analysis, Brooks Cole, Pacific Grove, CA, USA.

Paucean, A., Moldovan, O. P., Mureșan, V., Socaci, S. A., Dulf, F. V., Alexa, E., Man, S. M., Mureșan, A. E., and Muste, S. (2018). "Folic acid, minerals, amino-acids, fatty acids and volatile compounds of green and red lentils. Folic acid content optimization in wheat-lentils composite flours," Chemistry Central Journal 12(1), Article Number 88. DOI: $10.1186 / \mathrm{s} 13065-018-0456-8$

Phiri, C. (2010). "Influence of Moringa oleifera leaf extracts on germination and early seedling development of major cereals," Agriculture and Biology Journal of North America 1(5), 774-777. DOI: 10.5251/abjna.2010.1.5.774.777

Rai, V. K. (2002). "Role of amino acids in plant responses to stresses," Biologia Plantarum 45(4), 481-487. DOI: 10.1023/A:1022308229759

Razavi, F., Mahmoudi, R., Rabiei, V., Aghdam, M. S., and Soleimani, A. (2018). "Glycine betaine treatment attenuates chilling injury and maintains nutritional quality of hawthorn fruit during storage at low temperature," Scientia Horticulturae 233, 188-194. DOI: 10.1016/j.scienta.2018.01.053

Rizwan, M., Ali, S., Hussain, A., Ali, Q., Shakoor, M. B., Zia-Ur-Rehman, M., Farid, M. and Asma, M. (2017). "Effect of zinc-lysine on growth, yield and cadmium uptake in wheat (Triticum aestivum L.) and health risk assessment," Chemosphere 187, 35-42. DOI: $10.1016 /$ j.chemosphere.2017.08.071.

Ruberto, G., Renda, A., Daquino, C., Amico, V., Spatafora, C., Tringali, C., and De Tommasi, N. (2007). "Polyphenol constituents and antioxidant activity of grape pomace extracts from five Sicilian red grape cultivars," Food Chemistry 100(1), 203210. DOI: 10.1016/j.foodchem.2005.09.041

Sadak, M., Abdelhamid, M. T., and Schmidhalter, U. (2015). "Effect of foliar application of aminoacids on plant yield and some physiological parameters in bean plants irrigated with seawater," Acta Biológica Colombiana 20(1), 141-152. DOI: 10.15446/abc.v20n1.42865

Sakr, W. R. A., El-Sayed, A. A., Hammouda, A. M., and El Deen, F. S. A. S. (2018). "Effect of NPK, aloe gel and moringa extracts on geranium plants," Journal of Horticultural Science \& Ornamental Plants 10(1), 1-16. DOI: 10.5829/idosi.jhsop.2018.01.16

Salem, M. Z. M., Ibrahim, I. H. M., Ali, H. M., and Helmy, H. M. (2020). “Assessment of the use of natural extracted dyes and pancreatin enzyme for dyeing of four natural 
textiles: HPLC analysis of phytochemicals," Processes 8(1), Article Number 59. DOI: $10.3390 / \mathrm{pr} 8010059$

Salem, M. Z. M., Mansour, M. M. A., and Elansary, H. O. (2019). "Evaluation of the effect of inner and outer bark extracts of sugar maple (Acer saccharum var. saccharum) in combination with citric acid against the growth of three common molds," Journal of Wood Chemistry and Technology 39(2), 136-147. DOI: 10.1080/02773813.2018.1547763

Sarmin, N. S. (2014). "Effect of Moringa oleifera on germination and growth of Triticum aestivum," Journal of Bioscience and Agriculture Research 2(2), 59-69. DOI: 10.18801/jbar.020214.20

Sekhon, B. S. (2003). "Chelates for micronutrient nutrition among crops," Resonance 8, 46-53. DOI: $10.1007 / \mathrm{BF} 02834402$

Shaaban, F. K. M., El-Hadidy, G. A. M., and Mahmoud, T. S. M. (2020). "Effects of salicylic acid, putrescine and moringa leaf extract application on storability, quality attributes and bioactive compounds of plum cv. 'Golden Japan'," Future of Food: Journal on Food, Agriculture and Society 8(2), 1-14. DOI: 10.17170/kobra202007201466

Shan, T., Jin, P., Zhang, Y., Huang, Y., Wang, X., and Zheng, Y. (2016). "Exogenous glycine betaine treatment enhances chilling tolerance of peach fruit during cold storage," Postharvest Biology and Technology 114(4), 104-110. DOI: 10.1016/j.postharvbio.2015.12.005

Siddhuraju, P., and Becker, K. (2003). "Antioxidant properties of various solvent extracts of total phenolic constituents from three different agroclimatic origins of drumstick tree (Moringa oleifera Lam.) leaves," Journal of Agricultural and Food Chemistry 51(8), 2144-2155. DOI: 10.1021/jf020444+

Souri, M. K. (2016). "Aminochelate fertilizers: The new approach to the old problem; a review," Open Agriculture 1(1), 118-123. DOI: 10.1515/opag-2016-0016

Souri, M. K., and Hatamian, M. (2019). "Aminochelates in plant nutrition: A review," Journal of Plant Nutrition 42(1), 67-78. DOI: 10.1080/01904167.2018.1549671

Souri, M. K., Naiji, M., and Aslani, M. (2018). "Effect of Fe-glycine aminochelate on pod quality and iron concentrations of bean (Phaseolus vulgaris L.) under lime soil conditions," Communications in Soil Science and Plant Analysis 49(2), 215-224. DOI: 10.1080/00103624.2017.1421655

Souri, M. K., Sooraki, F. Y., and Moghadamyar, M. (2017). "Growth and quality of cucumber, tomato, and green bean under foliar and soil applications of an aminochelate fertilizer," Horticulture, Environment, and Biotechnology 58(6), 530536. DOI: 10.1007/s13580-017-0349-0

Sparks, D. L., Page, A. L., Helmke, P. A., and Loeppert, R. H. (2016). Methods of Soil Analysis, Part 3: Chemical Methods, John Wiley \& Sons, Hoboken, NJ, USA.

Stakhova, L. N., Stakhov, L. F., and Ladygin, V. G. (2000). "Effects of exogenous folic acid on the yield and amino acid content of the seed of Pisum sativum L. and Hordeum vulgare L.," Applied Biochemistry and Microbiology 36, 85-89. DOI: 10.1007/BF02738142

Teixeira, W. F., Fagan, E. B., Soares, L. H., Umburanas, R. C., Reichardt, K., and Neto, D. D. (2017). "Foliar and seed application of amino acids affects the antioxidant metabolism of the soybean crop," Frontiers in Plant Science 8(327), 1-14. DOI: 10.3389/fpls.2017.00327 
Torres-Urrutia, C., Guzman, L., Schmeda-Hirschmann, G., Moore-Carrasco, R., Alarcon, M., Astudillo, L., ... and Palomo, I. (2011). "Antiplatelet, anticoagulant, and fibrinolytic activity in vitro of extracts from selected fruits and vegetables," Blood coagulation \& fibrinolysis 22(3), 197-205. DOI: 10.1097/MBC.0b013e328343f7da

Turner, A. D., Hatfield, R. G., Rapkova, M., Higman, W., Algoet, M., Suarez-Isla, B. A., and Lees, D. N. (2011). "Comparison of AOAC 2005.06 LC official method with other methodologies for the quantitation of paralytic shellfish poisoning toxins in UK shellfish species," Analytical and Bioanalytical Chemistry 399(3), 1257-1270. DOI 10.1007/s00216-010-4428-7

Wang, H., Pampati, N., McCormick, W. M., and Bhattacharyya, L. (2016). "Protein nitrogen determination by Kjeldahl digestion and ion chromatography," Journal of Pharmaceutical Sciences 105(6), 1851-1857. DOI: 10.1016/j.xphs.2016.03.039

Wang, Y., Gao, Y., Ding, H., Liu, S., Han, X., Gui, J., and Liu, D. (2017). "Subcritical ethanol extraction of flavonoids from Moringa oleifera leaf and evaluation of antioxidant activity," Food chemistry 218, 152-158. DOI: 10.1016/j.foodchem.2016.09.058

Weiwei, C., Jinrong, L., Fang, X., and Jing, L. (2017). "Improvement to the determination of activated phosphorus in water and wastewater by yellow vanadomolybdate method," Industrial Water Treatment 37(2), 95-97. DOI: 10.11894/1005-829x.2017.37(2).095

Zobiole, L.H.S., de Oliveira Junior, R.S., Constantin, J., Kremer, R.J. and Biffe, D.F. (2012). "Amino acid application can be an alternative to prevent glyphosate injury in glyphosate-resistant soybeans", Journal of plant nutrition 35(2), 268-287. DOI: 10.1080/01904167.2012.636130

Article submitted: January 18, 2021; Peer review completed: March 6, 2021; Revised version received: March 12, 2021; Accepted: March 14, 2021; Published: March 22, 2021.

DOI: $10.15376 /$ biores.16.2.3391-3410 